\title{
Reply to Review of Neither Brain nor Ghost
}

\section{Teed Rockwell}

Professor McCarthy ${ }^{1}$ has carefully read many passages in my book Neither Brain nor Ghost (NBG), and clearly understands many of my main points. Unfortunately, there are other passages throughout the book that she seems to have missed, which contradict many of her criticisms. However, I have heard many of these criticisms before, so I probably have not been clear enough on these points. I welcome the opportunity to make further clarifications.

I could respond to her objection that no one believes that the brain possesses intrinsic causal powers by referring to the quotes from Jerry Fodor and Colin McGinn on pages 59 and 60 . But my main point was that these kinds of concepts are the only way to make sense out of metaphors like Dennett's claim that "the Head is Headquarters" or Paul Churchland's description of the brain as "the seat of the soul." There are a variety of causal factors that are responsible for the experiences we have. These include brain activity, other kinds of body activity, light bouncing of objects, and the objects themselves. Why is it that we assume that the activities within the brain "embody" experience, while these other factors only "cause" the experience? Why is it that only causal activity within the skull is given this special honorific? The answer seems obvious until you ask the question. Once the question is asked, the only answers available are the sort that McCarthy says no one would accept: that the brain is autonomous, or a closed system, or has intrinsic causal powers which make it fully responsible for the production of mental states. There are quotes, in my book and elsewhere, which show that many philosophers and scientists do accept these answers. But the important point is that no other available answer will support the claim that the mind is identical to the brain, regardless of who acknowledges this fact. To some degree my book is a challenge to those who defend mind/brain identity theory. "If you don't use these criteria for determining mind/brain identity, what criteria should we use?" My claim is that these kinds of criteria, weak as they are, remain the only reasons we have for believing that the mind is identical to the brain.

McCarthy frets about my "insouciance about the intricate processes in the brain that are necessarily involved in the generation of the conscious experience of any organism." I felt no need to add my voice to the chorus of wonder about the brain's intricacies, but that was not meant to imply disagreement. I felt a greater need to deal with those marginalized biological facts about consciousness and cognition 
which are not explainable solely by talking about the brain, because most people don't know about them, and because I believe they are philosophically important. (They appear mostly in chapters 2, 3 and 10.) More importantly, as I explain on page 30 , the fact that these brain processes are necessary for conscious experience does not mean that they are identical to conscious experience. Mind-Brain Identity requires that those brain processes be both necessary and sufficient. It is necessary for your car to have spark plugs in order for you to drive it. But that doesn't mean the spark plugs are identical to the car, because owning a set of spark plugs is not sufficient to enable you to drive to work. So the question remains: Why is brain activity identical to consciousness, and not just necessary for it? At what point does the intricacy become both necessary and sufficient, and why should we automatically assume that all of this intricacy will take place in the skull?

When most people get to this stage in the argument, they usually reply, as McCarthy did, that, even if we can't answer that question, we still need to make a distinction between Mind and World for pragmatic reasons. She, Dewey and I all agree that there is value in what McCarthy calls the "analytic distinctions of self and world." That is why I say this on page 104 of NBG: "To say that the mind emerges from the brain-body-world nexus does not mean that there is no world, only a mind. The line between the self and the world must always be drawn somewhere. ... That is what it means to live in a world." I do not identify the mind with the entire brain-body-world nexus, as McCarthy claims. I think what prompted her to attribute this position to me was the qualifiers that were removed from the above quote. I believe that the line between the self and world must be drawn somewhere at any given moment. But this does not necessarily imply that there is a single place that the line can be drawn for all conscious creatures, or for a single conscious creature throughout its history. A great deal of useful scientific work can be done by drawing the line at the skull, but the scientific work I describe in my book needs to draw the line in a variety of other places. I think the best way to account for both mainstream neuroscience and this other, more problematic work is to see the boundary between self and world as flexible. That is why I feel the mind is best described as a "behavioral field" rather than as an organ in the skull.

There is nothing mystical about this claim. Gravitational fields are every bit as physical as falling apples, but our best science cannot fully explain the behavior of falling apples without talking about fluctuations with a field. I am suggesting that if our psychology ever becomes as sophisticated as our physics, we might end up describing minds as similarly fluctuating behavioral fields. We can ignore these fluctuations a great deal of the time. The assumption that the mind is nothing but an organ in the skull will probably always be accurate enough for a variety of scientific research, just as Einsteinian physics is not necessary for most engineering jobs. But I believe that the future of cognitive science, and the resolution of certain philosophical puzzles, will require a subtler and initially more counterintuitive theory.

I could (and will) quibble about some of McCarthy's other criticisms. She implies that I am not aware that Searle's "Background" is a set of non-intentional 
capacities, although I devote pages 141-148 to explaining why Searle disagrees with both Dewey and me on this issue. She then claims that I have no right to use my amended version of Searle's concept because he is an internalist and I am an externalist, but gives no support for this. McCarthy also accuses me of having "limited acquaintance with requisite scientific knowledge," an accusation that is probably true of me and of anyone else who has written a multidisciplinary work of this sort. However, she does not cite a single factual error in support of this accusation. If she knows of some, I would like to hear about them. She also says that my criticism of Searle's distinction between intrinsic and observer-relative properties rests entirely on a few comments about the nature of entropy and energy, although the argument is actually three pages long, and makes several other points. Finally, Searle's distinction between brute and institutional facts is not limited to questions of syntax, as she claims, but includes all properties that presuppose the existence of purpose, including biological properties like being a lung.

However, my intention has been not so much to criticize Professor McCarthy's criticism as to make my own position clearer. I appreciate her willingness to question and challenge what is still very much a theory in progress, and I hope my replies will make it easier for future development and understanding of these new ideas.

\section{Note}

1. Christine McCarthy, "Book Review: Neither Brain nor Ghost: A Nondualist Alternative to the Mind-Brain Identity Theory," Education \& Culture 22, no. 2 (2006): 83-86; http://docs.lib.purdue.edu/eandc/vol22/iss2/art8.

Teed Rockwell is a lecturer at Somona State University.

Email:mcmf@california.com 